\title{
BMJ Open COVID-19 outcomes among adult patients treated with long-term opioid therapy for chronic non-cancer pain in the USA: a retrospective cohort study
}

Wen-Jan Tuan (D) , Hannah Spotts, Aleksandra E Zgierska, Robert P Lennon (D)

To cite: Tuan W-J, Spotts H, Zgierska AE, et al. COVID-19 outcomes among adult patients treated with long-term opioid therapy for chronic non-cancer pain in the USA: a retrospective cohort study. BMJ Open 2021;11:e056436. doi:10.1136/ bmjopen-2021-056436

- Prepublication history and additional supplemental material for this paper are available online. To view these files, please visit the journal online (http://dx.doi.org/10.1136/ bmjopen-2021-056436)

Received 14 August 2021 Accepted 27 0ctober 2021

Check for updates

(C) Author(s) (or their employer(s)) 2021. Re-use permitted under CC BY-NC. No commercial re-use. See rights and permissions. Published by BMJ.

Department of Family and Community Medicine, Penn State College of Medicine, Hershey, Pennsylvania, USA

Correspondence to Dr Wen-Jan Tuan; wtuan@pennstatehealth.psu. edu

\section{ABSTRACT}

Objective Patients treated with long-term opioid therapy (LTOT) are known to have compromised immune systems and respiratory function, both of which make them particularly susceptible to the SARS-CoV-2 virus. The objective of this study was to assess the risk of developing severe clinical outcomes among COVID-19 non-cancer patients on LTOT, compared with those without LTOT. Design and data sources $A$ retrospective cohort design using electronic health records in the TriNetX research database.

Participants and setting 418216 adults diagnosed with COVID-19 in January-December 2020 from 51 US healthcare organisations: 9558 in the LTOT and 408658 in the control cohort. They did not have cancer diagnoses; only a small proportion might have been treated with opioid maintenance for opioid use disorder.

Results Patient on LTOT had a higher risk ratio (RR) than control patients to visit an emergency department (RR 2.04, 95\% Cl 1.93 to 2.16) and be hospitalised (RR 2.91, $95 \% \mathrm{Cl} 2.69$ to 3.15). Once admitted, LTOT patients were more likely to require intensive care (RR $3.65,95 \% \mathrm{Cl}$ 3.10 to 4.29 ), mechanical ventilation (RR $3.47,95 \% \mathrm{Cl}$ 2.89 to 4.15 ) and vasopressor support (RR $5.28,95 \% \mathrm{Cl}$ 3.70 to 7.53 ) and die within 30 days (RR $1.96,95 \% \mathrm{Cl}$ 1.67 to 2.30). The LTOT group also showed increased risk (RRs from 2.06 to 3.98 , all significant to $95 \% \mathrm{Cl}$ ) of moresevere infection (eg, cough, dyspnoea, fever, hypoxaemia, thrombocytopaenia and acute respiratory distress syndrome). Statistically significant differences in several laboratory results and other vital signs appeared clinically negligible.

Conclusion COVID-19 patients on LTOT were at higher risk of increased morbidity, mortality and healthcare utilisation. Interventions to reduce the need for LTOT and to increase compliance with COVID-19 protective measures may improve outcomes and reduce healthcare cost in this population. Prospective studies need to confirm and refine these findings.

\section{INTRODUCTION}

The COVID-19 pandemic, resulting from SARS-CoV-2 infection, has rapidly spread across the United States since early 2020. By the end of 2020, there were over 20 million confirmed cases and 344000 deaths reported

\section{Strengths and Limitations of this Study}

- This study used large-scale electronic health recordbased data and propensity score matching to assess the risk of morbidity and mortality from SARS-CoV-2 infection among US adults treated with long-term opioids for chronic non-cancer pain.

- The study findings can help shape the conversations between clinicians, public health personnel and patients on optimal prevention and early treatment protocols for safer and more effective long-term opioid therapy.

- As a retrospective cohort study, the analysis may be missing data from patients tested or treated for COVID-19 infection outside the research data network, potentially skewing results.

- The study did not assess associations between the dosage of prescribed opioids and the outcomes in patients with COVID-19 illness.

in the USA. ${ }^{1}$ This unprecedented upheaval has led to deaths from the novel coronavirus, in addition to deaths caused by the effects of protracted economic stagnation and social disruption. Vulnerable populations with mental illness or substance use disorders have been disproportionately affected. ${ }^{23}$ As the nation focuses on the COVID-19 pandemic, the opioid crisis has continued to have devastating impacts on communities. Recent statistics show a $38.4 \%$ increase in opioid-related deaths from June 2019 to May 2020, ${ }^{4}$ and state-level data have linked stressors of the COVID-19 pandemic to surge in fatal overdoses. ${ }^{5}$ Literature suggests the opioid crisis has been escalated by a lack of access to drug screening and treatment for opioid use disorder (OUD) due to care disruption by the COVID-19 pandemic. ${ }^{6}$ Ongoing opioid addiction prevention efforts have also been disrupted by social distancing practices and isolation that can contribute to the misuse of prescription or illicit opioids. ${ }^{37}$ However, studies focused on the opioid crisis in the 
United States often look only at persons with substance abuse disorders, who have numerous comorbidities with independent COVID-19 risk; little is known about the impact of COVID-19 on people on long-term opioid therapy (LTOT) who do not have such disorders, but may be at increased COVID-19 risk by virtue of their LTOT alone.

Research shows that opioids can trigger acute respiratory depression (eg, hypoventilation and hypoxaemia) through the activation of opioid receptors in the brainstem can lead to respiratory failure or death. ${ }^{8}$ Chronic opioid use also increases the risk of immunosuppression and infections, including among people on LTOT. ${ }^{9}$ These individuals are likely to have cardiopulmonary morbidity, longer hospitalisation, and greater overall care costs. With severe COVID-19 infection, patients may also present with clinical signs and symptoms of respiratory depression. ${ }^{10}{ }^{11}$ Approximately $10 \%-15 \%$ of patients hospitalised for COVID-19 progressed to acute respiratory distress syndrome (ARDS).${ }^{12}{ }^{13}$ While the risk of increasing morbidity and mortality from SARS-CoV-2 infection among individuals with certain health conditions has been identified and incorporated into outcome prediction models, the relationship between LTOT and SARS-CoV-2-related morbidity and mortality has not been assessed. ${ }^{14}$ The likelihood of worsened outcomes in patients on LTOT and with COVID-19 may be caused by the mechanisms of respiratory depression and immunosuppression. ${ }^{15}$ As a result, this patient population may be expected to have more severe health outcomes, potentially resulting in an increased risk of hospitalisation, emergency department (ED) admissions and time in the intensive care unit (ICU). ${ }^{16}{ }^{17}$ Critically ill patients with SARS-CoV-2 were also more likely to be treated with mechanical ventilation and vasopressors. ${ }^{18} 19$ Although COVID-19 outcomes are known to be worse in persons with opioid and other substance use disorders, ${ }^{20}$ such disorders may be clinically underdiagnosed. Further, the vast majority of persons prescribed opioids for chronic pain do not have substance use disorders, ${ }^{21}$ yet may still be at risk from their LTOT alone. Hence, research is urgently needed to investigate long-term opioid use in populations beyond those with substance use disorders as a pathway to severe COVID-19.

This study aims to assess the risk of developing severe outcomes among adults with LTOT for chronic noncancer pain and with COVID-19 infection in the USA in order to help clinicians develop more effective care guidelines for patients with COVID-19 and raise awareness about the risks of COVID-19 to vulnerable populations.

\section{METHODS}

\section{Study design and data collection}

The study applied a retrospective cohort design using electronic health records (EHRs) from 51 healthcare organisations, members of the research network of the TriNetX database in the USA (Cambridge, Massachusetts,
USA). TriNetX is a federated health research network that provides access to continuously updated, deidentified EHR data (demographics, diagnoses, procedures, medications, laboratory tests and genomics) of more than 68 million patients from participating healthcare organisations. The TriNetX platform only uses aggregated counts and statistical summaries.

\section{Cohort description}

The study population consisted of adults (age $\geq 18$ years) a diagnosis of COVID-19 between 1 January 2020 and 31 December 2020, based on the combination of one or more disease indicators, including the International Classification of Diseases, 10th Edition (ICD-10) diagnosis codes or positive laboratory test results. Individuals are considered on LTOT when they are prescribed with opioids in three or more consecutive months or at least 90 days at outpatient settings. ${ }^{22}{ }^{23}$ COVID-19 patients meeting the LTOT criteria within 12 months before their infection were assigned to the LTOT cohort. Individuals with COVID-19 without LTOT in the past 12 months were assigned to the control cohort. The analysis excluded individuals who had cancers, or living in nursing home, hospice or palliative care facilities.

Moreover, this study included various types of opioid analgesics prescribed in outpatient settings, and targeted the population of adults with chronic non-cancer pain. We excluded patients who had cancer diagnoses to limit the impact of opioids prescribed for cancer pain. Although methadone and buprenorphine can be used to treat OUD in addition to chronic pain, methadone prescriptions issued in the outpatient settings are exclusively for pain care; only specialised opioid treatment programmes can use methadone for OUD care by dispensing it, thus, this clinical application would not have been captured in our dataset. Buprenorphine can be prescribed for OUD in the outpatient settings for both chronic pain and OUD indications; the research dataset did not allow us to determine the specific indication; we elected to retain those treated with buprenorphine because buprenorphine could have been prescribed to treat chronic pain. In addition, only a small proportion of the study sample was treated with buprenorphine $(5.0 \%)$, with even smaller proportion $(1.6 \%)$ having both the OUD diagnosis and being prescribed buprenorphine during the study period.

\section{Outcome indicators}

The severity of the COVID-19 infection was assessed through three areas: healthcare utilisation, clinical presentation and diagnostic testing. The healthcare utilisation and mortality measures included binary variables (yes/no) indicating whether patients were admitted to $\mathrm{ED}$, inpatient hospital, ICU, placed on mechanical ventilation, treated with vasopressors or died within 30 days after being infected by COVID-19. Similarly, the clinical presentation measures also indicated the presence/ absence (yes/no) of severe physical signs or medical complication, including cough, fever, ARDS, hypoxaemia, 
thrombocytopaenia and dyspnoea. The diagnostic testing consisted of common biometrics or laboratory tests serving as severity indicators of COVID-19 infection, such as $\mathrm{C}$ reactive protein (CRP), serum creatinine and blood urea nitrogen (BUN). These tests have also been used to predict the risk of increased COVID-19-related morbidity and mortality in both inpatient and outpatient settings. ${ }^{14} 24$

\section{Data analysis}

Data in the TriNetX database have been shown referential integrity and be reliable. ${ }^{25}$ The coding information of the research data also underwent extensive curation and was mapped to common clinical terminologies to ensure high usability and consistency with the Reporting of studies Conducted using Observational Routinely collected Data guidelines criteria. ${ }^{26} \mathrm{~A}$ number of patient characteristics were considered potential confounding variables, including age, sex, race/ethnicity and comorbidities (diabetes, essential hypertension, chronic pulmonary conditions, cardiovascular diseases, chronic kidney diseases, mental health disorders). To address potential confounding effects of the socioeconomic status, we included diagnoses, which may indicate increased risk due to socioeconomic and psychosocial circumstances (education and literacy, employment, housing, lack of adequate food or water or exposure to occupational hazards). The study applied a 1:1 propensity score matching (PSM) technique to balance the baseline characteristics between the comparison and control cohorts, and reduce potential selection bias. The matching method was performed using nearest neighbour algorithms with a calliper width of 0.1 pooled SD. Outcomes were compared in COVID-19 patients on LTOT and COVID-19 patients not on LTOT using logistic regression modelling before and after PSM. Risk ratios (RR), with $95 \%$ CIs were computed and a twosided alpha of less than 0.05 was defined a priori for statistical significance between the two groups. All data queries and statistical analyses were performed using build-in analytics functions on the TriNetX portal. Detailed information for the diagnoses and laboratory tests are provided in online supplemental table 1.

\section{Patients and public involvement statement}

Neither patients nor the public were involved in the design, or conduct, or reporting, or dissemination plans of our research.

\section{RESULTS}

\section{Study population}

A total of 418216 patients diagnosed with COVID-19 from 51 healthcare organisations met the study eligibility criteria, including 9558 individuals in the LTOT cohort and 408658 in the non-LTOT cohort. Before PSM, the LTOT cohort was older, with a greater percentage of female, white and black patients compared with the control cohort (see table 1).
The LTOT cohort had a greater proportion of males and patients with an unidentified race, and consistently higher prevalence of comorbidities than their non-LTOT counterparts. After PSM, most of these differences became not significant, suggesting the demographic characteristics and comorbid conditions were well-balanced between the LTOT and non-LTOT cohorts. Moreover, absolute standardised differences for all measured baseline characteristics were less than $10 \%$, further confirming that both cohorts had similar distributions of the observed baseline characteristics and matched samples. ${ }^{27}$

\section{Healthcare utilisation and mortality}

Before the PSM, COVID-19 patients on LTOT were more likely to visit ED (RR 3.80; 95\% CI 3.67 to 3.92) and be hospitalised (RR 6.62; 95\% CI 6.36 to 6.90 ) than individuals without LTOT. They also were more likely to receive intensive care (RR 9.03; 95\% CI 8.33 to 9.80), mechanical ventilation (RR 7.75; 95\% CI 7.07 to 8.50 ) and vasopressors (RR 10.42; 95\% CI 8.90 to 12.20) and were more likely to die within 30 days post-COVID-19 diagnosis (RR 4.04; $95 \%$ CI 8.90 to 12.20 ), compared with their non-LTOT counterparts. After PSM, the adjusted risk of using inpatient care resources or extensive life support remained 2.0-5.3 times higher for patients on LTOT compared with the control cohort (all significant to 95\% CI) (see figure 1 ). The 30 -day postdiagnosis mortality rates were also found to be consistently higher in the LTOT cohort, regardless of the PSM adjustment (RR 1.96; 95\% CI 1.67 to 2.30) (see online supplemental table 2) for details of our results before and after PSM .

\section{Clinical presentation}

In prematching analysis, patients on LTOT were three times more likely $(p<0.01)$ to have fever and/or cough than their non-LTOT counterparts. The LTOT cohort also showed greater risk of ARDS (RR 3.98; 95\% CI 2.91 to 5.44), hypoxaemia (RR 2.41; $95 \%$ CI 2.10 to 2.76), dyspnoea (RR 2.18; 95\% CI 2.03 to 2.35) and thrombocytopaenia (RR 2.28; $95 \%$ CI 1.84 to 2.84). In the post-PSM analyses, patients on LTOT were consistently found to have more medical complications compared with nonLTOT patients (see figure 2). The adjusted RRs (all significant to $95 \% \mathrm{CI}$ ) were 2.06 for cough, 2.24 for fever, 2.18 for dyspnoea, 2.28 for thrombocytopaenia, 2.41 for hypoxaemia and 3.98 for ARDS. See online supplemental table 3 for details of our results before and after PSM.

\section{Laboratory tests}

Mixed results were found in vital and laboratory tests commonly ordered to assess the severity of COVID-19 in the pre-PSM and post-PSM analyses (see table 2). Higher systolic blood pressure (126.9 vs 124.3 , p<0.01) was observed among LTOT patients before PSM, while there were no significant differences in lower systolic (126.7 vs 127.6, $\mathrm{p}=0.09$ ) and diastolic ( 74.7 vs $75.1, \mathrm{p}=0.12$ ) blood pressure values between LTOT and non-LTOT patients after matching. 
Table 1 Patient demographics and comorbidities, before and after propensity score matching

\begin{tabular}{|c|c|c|c|c|c|c|c|}
\hline \multirow[b]{2}{*}{ Characteristic } & \multicolumn{3}{|c|}{ Before propensity score matching } & \multicolumn{3}{|c|}{ After propensity score matching } & \multirow[b]{2}{*}{$\begin{array}{l}\text { Standardised } \\
\text { mean } \\
\text { difference }\end{array}$} \\
\hline & $\begin{array}{l}\text { Long-term } \\
\text { opioid therapy } \\
\text { ( } \mathrm{N}=9558)\end{array}$ & $\begin{array}{l}\text { No long-term } \\
\text { opioid therapy } \\
\text { ( } \mathrm{N}=408 \text { 658) }\end{array}$ & P value & $\begin{array}{l}\text { Long-term } \\
\text { opioid therapy } \\
\text { ( } \mathrm{N}=9558 \text { ) }\end{array}$ & $\begin{array}{l}\text { No long-term } \\
\text { opioid therapy } \\
\text { ( } \mathrm{N}=9558 \text { ) }\end{array}$ & P value & \\
\hline Age, mean $\pm S D$ & $52.1 \pm 17.1$ & $43.1 \pm 17.6$ & $<0.001$ & $52.1 \pm 17.1$ & $52.7 \pm 17.7$ & 0.063 & 0.033 \\
\hline \multicolumn{8}{|l|}{ Sex } \\
\hline Female, n (\%) & $5793(60.6)$ & 208267 (51) & $<0.001$ & $5793(60.6)$ & $5743(60.1)$ & 0.460 & 0.011 \\
\hline Male, n (\%) & 3764 (39.4) & 199947 (48.9) & $<0.001$ & $3764(39.4)$ & $3804(39.8)$ & 0.554 & 0.009 \\
\hline \multicolumn{8}{|l|}{ Hispanic or Latino } \\
\hline Yes, n (\%) & $1384(14.5)$ & $50634(12.4)$ & $<0.001$ & $1384(14.5)$ & $1320(13.8)$ & 0.184 & 0.019 \\
\hline No, n (\%) & $6017(63)$ & $178818(43.8)$ & $<0.001$ & $6017(63)$ & $5995(62.7)$ & 0.742 & 0.005 \\
\hline \multicolumn{8}{|l|}{ Race } \\
\hline White, n (\%) & $5969(62.5)$ & 212907 (52.1) & $<0.001$ & $5969(62.5)$ & $6045(63.2)$ & 0.255 & 0.016 \\
\hline $\begin{array}{l}\text { Black or African } \\
\text { American, } \mathrm{n}(\%)\end{array}$ & $2200(23)$ & $62396(15.3)$ & $<0.001$ & $2200(23)$ & $2234(23.4)$ & 0.560 & 0.008 \\
\hline Unknown, n (\%) & $1128(11.8)$ & $120420(29.5)$ & $<0.001$ & $1128(11.8)$ & $1029(10.8)$ & 0.094 & 0.033 \\
\hline $\begin{array}{l}\text { Essential (primary) } \\
\text { hypertension, n (\%) }\end{array}$ & $4816(50.4)$ & $39656(9.7)$ & $<0.001$ & $4816(50.4)$ & $4983(52.1)$ & 0.166 & 0.035 \\
\hline $\begin{array}{l}\text { Chronic lower } \\
\text { respiratory diseases, } \\
\mathrm{n}(\%)\end{array}$ & $2425(25.4)$ & $19849(4.9)$ & $<0.001$ & $2425(25.4)$ & $2561(26.8)$ & 0.250 & 0.032 \\
\hline $\begin{array}{l}\text { Diabetes mellitus, } \mathrm{n} \\
(\%)\end{array}$ & $2682(28.1)$ & $18589(4.5)$ & $<0.001$ & $2682(28.1)$ & 2639 (27.6) & 0.488 & 0.010 \\
\hline $\begin{array}{l}\text { Overweight and } \\
\text { obesity, } \mathrm{n}(\%)\end{array}$ & 3089 (32.3) & $23383(5.7)$ & $<0.001$ & 3089 (32.3) & 3171 (33.2) & 0.206 & 0.018 \\
\hline $\begin{array}{l}\text { Ischaemic heart } \\
\text { diseases, } n(\%)\end{array}$ & 1575 (16.5) & $7336(1.8)$ & $<0.001$ & 1575 (16.5) & $1422(14.9)$ & 0.020 & 0.044 \\
\hline Heart failure, n (\%) & 1176 (12.3) & $3865(0.9)$ & $<0.001$ & $1176(12.3)$ & $925(9.7)$ & $<0.001$ & 0.084 \\
\hline $\begin{array}{l}\text { Chronic kidney } \\
\text { disease, n (\%) }\end{array}$ & $1448(15.2)$ & $5123(1.3)$ & $<0.001$ & $1448(15.2)$ & 1294 (13.5) & $<0.001$ & 0.046 \\
\hline $\begin{array}{l}\text { Nicotine dependence, } \\
\mathrm{n}(\%)\end{array}$ & 1232 (12.9) & 8937 (2.2) & $<0.001$ & $1232(12.9)$ & $1248(13.1)$ & 0.73 & 0.005 \\
\hline $\begin{array}{l}\text { Alcohol related } \\
\text { disorders, n (\%) }\end{array}$ & $430(4.5)$ & $2848(0.7)$ & $<0.001$ & $430(4.5)$ & $426(4.5)$ & 0.889 & 0.002 \\
\hline $\begin{array}{l}\text { Socioeconomic } \\
\text { circumstances, n (\%) }\end{array}$ & $469(4.9)$ & $2772(0.7)$ & $<0.001$ & $469(4.9)$ & $464(4.9)$ & 0.867 & 0.002 \\
\hline
\end{tabular}

$\mathrm{n}$, number of patients.

The prematching analysis further showed that the LTOT cohort had a lower concentration of serum ferritin (613.8 vs $742.8, \mathrm{p}<0.01)$ and lower platelet count $(259.7$ vs $263.9, \mathrm{p}<0.01)$, but differences in these lab results were not significantly different between the two cohorts in the postmatching analysis. Despite no difference found in the leucocyte count and ALT concentration before matching, elevation in leucocytes (8.1 vs 7.7, $\mathrm{p}<0.01)$ and ALT (40.4 vs 31.5, $\mathrm{p}<0.01$ ) were observed in the LTOT cohort after the matching, compared with the control cohort. Moreover, in both the prematching and postmatching analyses, patients on LTOT showed lower lymphocyte counts (23.5 vs $25.3, \mathrm{p}<0.01$ before matching; 23.5 vs $24.8, \mathrm{p}<0.01$ after matching), yet greater serum concentrations of creatinine, ALP and CRP than their non-LTOT counterparts (see table 2).

\section{DISCUSSION}

The COVID-19 pandemic has presented persistent public health challenges, particularly among populations with a history of substance use and mental health conditions. Amidst the pandemic, the crisis of the opioid epidemic has continued to rise and strain healthcare resources, society productivity and general well-being. ${ }^{16}{ }^{17}$ Yet, while the literature has identified the pernicious effects of COVID-19 on individuals with OUD,${ }^{6}{ }^{20}$ little is known about the outcomes and presentation of COVID-19 


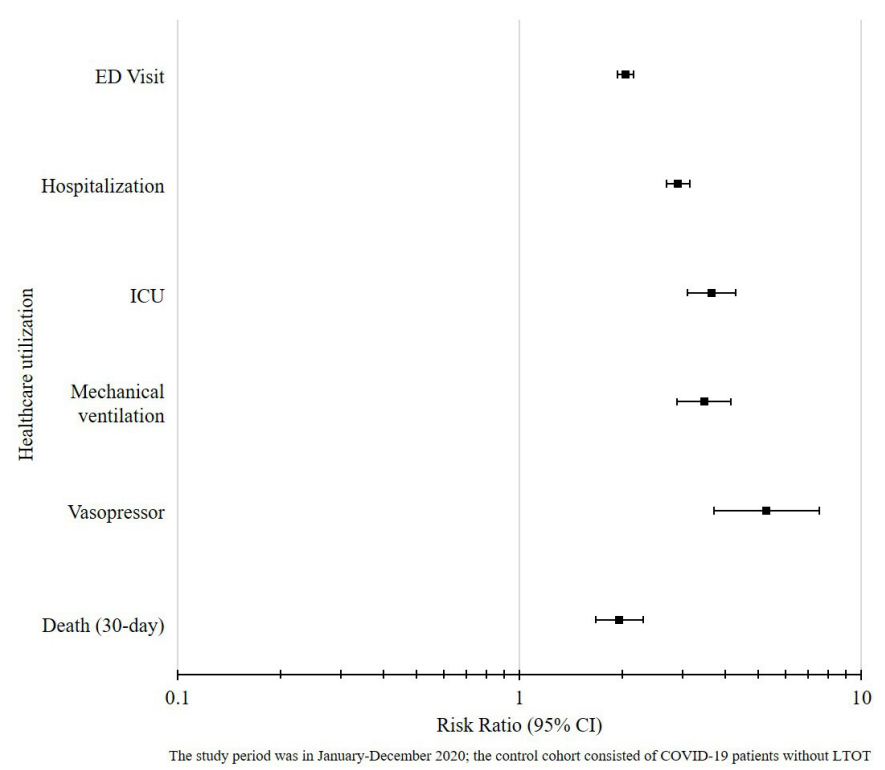

Figure 1 Healthcare utilization and mortality among COVID-19 patients with LTOT compared to COVID-19 patients without LTOT. ED, emergency department; ICU, intensive care unit; LTOT, long-term opioid therapy.

among patients treated with LTOT for chronic noncancer pain. Given the magnitude of both crises, lack of understanding of the relationship between COVID-19 and LTOT represents a gap, which can disadvantage clinicians when considering prevention and early treatment among individuals in this population.

This study revealed that COVID-19 patients with a history of LTOT were more likely to be admitted to the hospital, ED and ICU and have higher 30-day mortality rates. Additionally, there was greater use of both vasopressors and mechanical ventilation, suggesting that

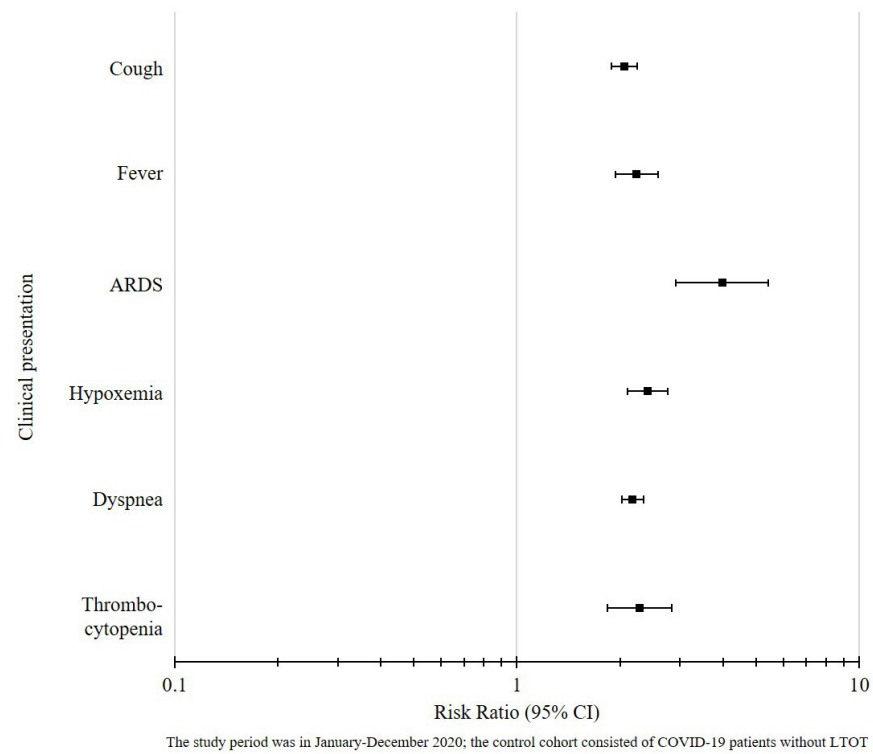

Figure 2 Clinical presentation among COVID-19 patients with LTOT compared to COVID-19 patients without LTOT. ARDS, acute respiratory distress syndrome; LTOT, long-term opioid therapy. long-term opioid users are more likely to get severely ill from COVID-19. This aligns with the existing literature that found the need for respiratory support in the ICU among COVID-19 patients struggling with hypoxaemia. ${ }^{28}$ Previous studies have shown more hospitalisations, ICU admissions and death among COVID-19 patients with any form of substance use disorder, with particularly strong associations among patients with OUD. ${ }^{20} 2930$ Our study demonstrates that patients on LTOT, which was primarily for chronic non-cancer pain, are also at increased risk of severe symptoms such as cough, fever, hypoxaemia, dyspnoea thrombocytopaenia and ARDS. There is significant overlap between the ways in which the pathophysiology of COVID-19 and the interactions of opioids with their $\mu$-receptors mediate both respiratory damage and immunosuppression. ${ }^{101131}$ As such, opioids can contribute to a decrease in cytokine and leucocyte recruitment, compromising the innate and adaptive immune pathways, potentially making individuals more susceptible to infection at the same time as opioidinduced respiratory depression amplifies hypoxaemia in COVID-19. ${ }^{915}$ While there is conflicting literature on the direct effects of opioids on cardiovascular events such as myocardial infarction, some research has demonstrated how cardiorespiratory co-morbidities play a role in the increased risk of severe outcomes among COVID-19 patients with OUD. ${ }^{30} 32$

Several studies have also examined the prognostic value of various laboratory tests in the setting of severe COVID-19. There were significant differences in leucocytes, lymphocytes, serum creatinine, BUN, ALT, ALP and CRP in our results after PSM. Previous studies have shown that COVID-19 patients have demonstrated some degree of lymphopenia with or without leucopenia, alterations in neutrophil to lymphocyte ratios, mild decreases in platelets, and elevations in inflammatory markers such as CRP and erythrocyte sedimentation rate. ${ }^{113}$ In patients on prescription opioids, research has documented some elevations in CRP and altered platelet, lymphocyte and monocyte ratios. ${ }^{34} 35$ Elevations in kidney, liver and other systemic organ lab results may indicate the effects of COVID-19 on causing multisystem organ damage or failure. ${ }^{1130}$ However, the absolute difference between groups for each of the laboratory values is small, with doubtful clinical significance.

\section{Limitations}

This study has several limitations to note and consider. First, there is a possibility that patients on LTOT captured in the TriNetX research database received their COVID-19 diagnosis or laboratory testing at facilities outside of the participating networks, and therefore would not have been included in the analysis. Second, although ideally we would have been able to clearly delineate a population of patients with LTOT prescribed for chronic non-cancer pain, it is possible that patients included in our analysis could have had long-term opioids prescribed for cancer pain or for OUD. Our excursion criteria with cancer 


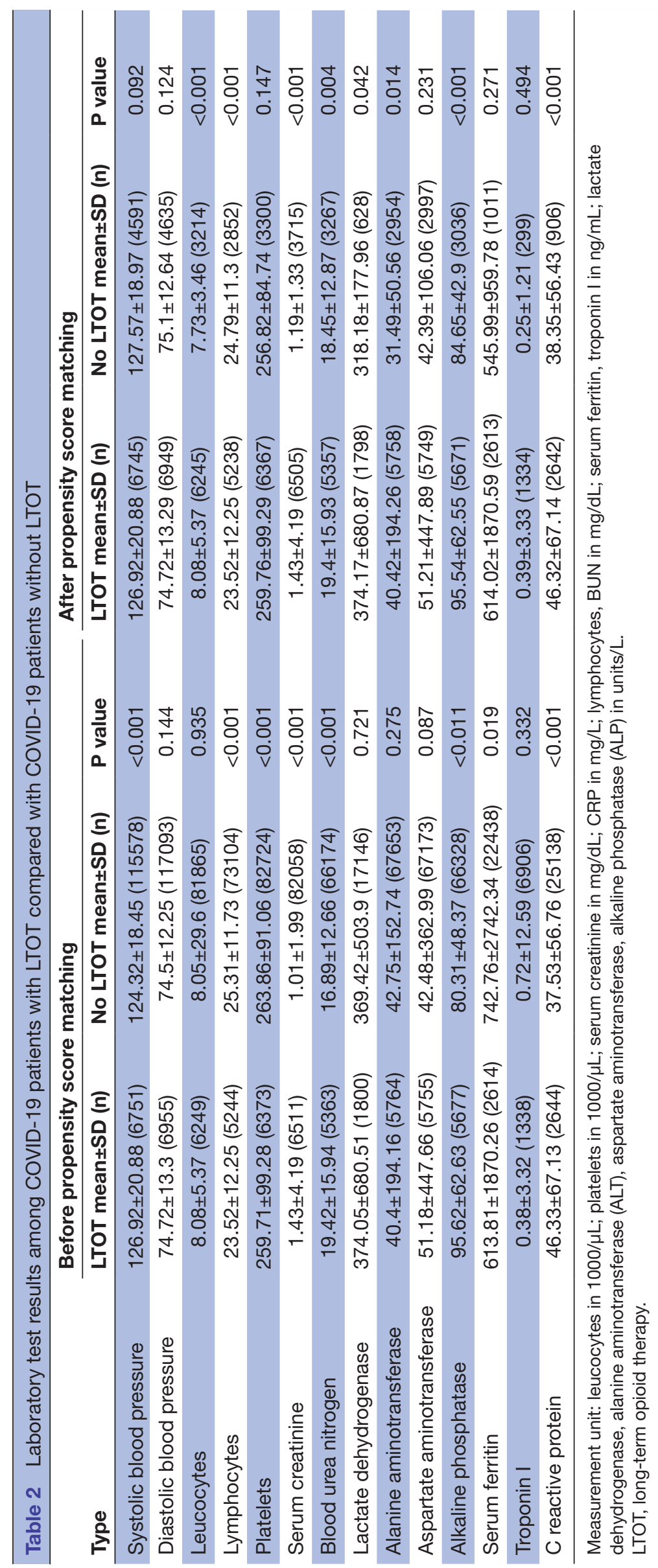


diagnoses and preliminary analyses used to estimate the proportion of patients with OUD diagnoses in our sample were designed to mitigate these impacts. Third, we were unable to account for the potential impact of opioid dose, because calculation of the daily morphineequivalent dose was not possible when using the available TriNetX data. We were also limited in our ability to determine the specific timing of opioid use in relation to the COVID-19 infection; the TriNetX data provided the information on opioid prescriptions issued within a specific time frame but this may not necessarily correspond to real-life use of opioids by patients; future research should implement a design, which could enable of better evaluation of timing/dose of opioids in relation to outcomes of interest. Fourth, there are several important socioeconomic factors that are not available in the research database, such as type of insurance, education and urban or rural residence that could act as confounders in the statistical analysis. However, a strength of the large sample size available allowed for robust PSM, which enabled us to construct comparable cohorts in order to best determine the LTOT effects on the selected outcomes and minimise the risk of confounders, increasing the generalisability of results. Lastly, there may be unobserved or unknown confounders present that we did not account for in propensity matching. Future analyses using advanced data mining techniques might be able to better identify currently unidentified yet important confounders.

\section{CONCLUSION}

This study leveraged EHR data available through a large national research database and suggested that LTOT is associated with increased risk of severe illness and complications, including death, in adults with COVID-19 infection. This is consistent with anticipated worse outcomes secondary to LTOT causing prolonged inflammation, acute respiratory distress, and ineffective immune responses. Efforts to decrease SARS-CoV-2 infection rates in persons on LTOT through personal mitigation behaviours (eg, masking, physical distancing, handwashing) and vaccination are critical to decrease morbidity. Further research, including prospective studies, is needed to confirm and refine these findings. These results suggest that efforts to decrease SARSCoV-2 infection rates in persons on LTOT (eg, through personal mitigation behaviours, such as masking, physical distancing, handwashing and through vaccination) and considering LTOT as a potential prognosticator for worse outcomes could be critical to decrease morbidity and mortality due to COVID-19 infections, particularly in this clinical population.

Contributors All authors were involved in revisions, read and approved the final manuscript. W-JT contributed to the planning and design of the work, literature review, data analysis, interpretation, writing the manuscript, and served as a guarantor responsible for the overall content. HS contributed to literature review, data analysis, interpretation and writing the manuscript. AEZ and RPL contributed to interpretation and writing the manuscript.
Funding The author(s) received no financial support for the research, authorship, and/or publication of this article. The work was conducted through the medical scholar research programme of the Penn State Department of Family and Community Medicine. Penn State Clinical and Translational Science Institute provides access to the TriNetX network and is supported by a National Centre for Advancing Translational Sciences and its Clinical and Translational Science Award (Grant: UL1 TR002014).

Competing interests None declared.

Patient consent for publication Not applicable.

Ethics approval Formal ethnical approval was not required. All the data queries were performed in the TriNetX online portal managed by the Penn State Clinic and Translational Science Institute. Because there was no protected health information data accessed in the analysis, this research was determined to be exempt from the institutional review board oversight.

Provenance and peer review Not commissioned; externally peer reviewed.

Data availability statement No data are available. No additional data are available.

Supplemental material This content has been supplied by the author(s). It has not been vetted by BMJ Publishing Group Limited (BMJ) and may not have been peer-reviewed. Any opinions or recommendations discussed are solely those of the author(s) and are not endorsed by BMJ. BMJ disclaims all liability and responsibility arising from any reliance placed on the content. Where the content includes any translated material, BMJ does not warrant the accuracy and reliability of the translations (including but not limited to local regulations, clinical guidelines, terminology, drug names and drug dosages), and is not responsible for any error and/or omissions arising from translation and adaptation or otherwise.

Open access This is an open access article distributed in accordance with the Creative Commons Attribution Non Commercial (CC BY-NC 4.0) license, which permits others to distribute, remix, adapt, build upon this work non-commercially, and license their derivative works on different terms, provided the original work is properly cited, appropriate credit is given, any changes made indicated, and the use is non-commercial. See: http://creativecommons.org/licenses/by-nc/4.0/.

\section{ORCID iDs}

Wen-Jan Tuan http://orcid.org/0000-0003-3939-8979

Robert P Lennon http://orcid.org/0000-0003-0973-5890

\section{REFERENCES}

1 Centers for Disease Control and Prevention. CDC COVID data tracker, 2021. Available: https://covid.cdc.gov/covid-data-tracker/\# trends_totalandratecases

2 Melamed OC, Hauck TS, Buckley L, et al. COVID-19 and persons with substance use disorders: inequities and mitigation strategies. Subst Abus 2020;41:286-91.

3 Ornell F, Moura HF, Scherer JN, et al. The COVID-19 pandemic and its impact on substance use: implications for prevention and treatment. Psychiatry Res 2020;289:113096.

4 Centers for Disease Control and Prevention. Overdose deaths accelerating during COVID-19, 2021. Available: https://www.cdc.gov/ media/releases/2020/p1218-overdose-deaths-covid-19.html

5 Macmadu A, Batthala S, Correia Gabel AM, et al. Comparison of characteristics of deaths from drug overdose before vs during the COVID-19 pandemic in Rhode island. JAMA Netw Open 2021;4:e2125538.

6 Khatri UG, Perrone J. Opioid use disorder and COVID-19: crashing of the crises. J Addict Med 2020;14:e6-7.

7 Silva MJ, Kelly Z. The escalation of the opioid epidemic due to COVID-19 and resulting lessons about treatment alternatives. Am J Manag Care 2020;26:e202-4.

8 Dahan A, Aarts L, Smith TW. Incidence, reversal, and prevention of opioid-induced respiratory depression. Anesthesiology 2010;112:226-38.

9 Wiese AD, Grijalva CG. The use of prescribed opioid analgesics \& the risk of serious infections. Future Microbiol 2018;13:849-52.

10 Grasselli G, Tonetti T, Protti A, et al. Pathophysiology of COVID19-associated acute respiratory distress syndrome: a multicentre prospective observational study. Lancet Respir Med 2020;8:1201-8.

11 Ragab D, Salah Eldin H, Taeimah M, et al. The COVID-19 cytokine storm; what we know so far. Front Immunol 2020;11:1446.

12 El-Solh AA, Meduri UG, Lawson Y, et al. Clinical course and outcome of COVID-19 acute respiratory distress syndrome: data from a national Repository. J Intensive Care Med 2021;36:664-72. 
13 Matthay MA, Aldrich JM, Gotts JE. Treatment for severe acute respiratory distress syndrome from COVID-19. Lancet Respir Med 2020;8:433-4.

14 Ebell $\mathrm{MH}$, Cai X, Lennon $\mathrm{R}$, et al. Development and validation of the COVID-NoLab and COVID-SimpleLab risk scores for prognosis in 6 US health systems. J Am Board Fam Med 2021;34:S127-35.

15 Schimmel J, Manini AF. Opioid use disorder and COVID-19: biological plausibility for worsened outcomes. Subst Use Misuse 2020;55:1900-1.

16 Bentivegna M, Hulme $\mathrm{C}$, Ebell $\mathrm{MH}$. Primary care relevant risk factors for adverse outcomes in patients with COVID-19 infection: a systematic review. J Am Board Fam Med 2021;34:S113-26.

17 Bravata DM, Perkins AJ, Myers LJ, et al. Association of intensive care unit patient load and demand with mortality rates in US department of Veterans Affairs hospitals during the COVID-19 pandemic. JAMA Netw Open 2021;4:e2034266.

18 loannou GN, Locke E, Green P, et al. Risk factors for hospitalization, mechanical ventilation, or death among 10131 US veterans with SARS-CoV-2 infection. JAMA Netw Open 2020;3:e2022310.

19 Poston JT, Patel BK, Davis AM. Management of critically ill adults with COVID-19. JAMA 2020;322:1839-41.

20 Wang QQ, Kaelber DC, Xu R, et al. COVID-19 risk and outcomes in patients with substance use disorders: analyses from electronic health records in the United States. Mol Psychiatry 2021;26:30-9.

21 Vowles KE, McEntee ML, Julnes PS, et al. Rates of opioid misuse, abuse, and addiction in chronic pain: a systematic review and data synthesis. Pain 2015;156:569-76.

22 Korff MV, Saunders K, Thomas Ray G, et al. De facto long-term opioid therapy for noncancer pain. Clin J Pain 2008;24:521-7.

23 Von Korff M, Kolodny A, Deyo RA, et al. Long-term opioid therapy reconsidered. Ann Intern Med 2011;155:325-8.

24 Galloway JB, Norton S, Barker RD, et al. A clinical risk score to identify patients with COVID-19 at high risk of critical care admission or death: an observational cohort study. J Infect 2020;81:282-8.
25 Topaloglu U, Palchuk MB. Using a Federated network of real-world data to optimize clinical trials operations. JCO Clin Cancer Inform 2018;30:1-10.

26 Benchimol El, Smeeth L, Guttmann A, et al. The reporting of studies conducted using observational Routinely-collected health data (record) statement. PLoS Med 2015;12:e1001885.

27 Morgan CJ. Reducing bias using propensity score matching. J Nucl Cardiol 2018;25:404-6.

28 Grasselli G, Zangrillo A, Zanella A, et al. Baseline characteristics and outcomes of 1591 patients infected with SARS-CoV-2 admitted to ICUs of the Lombardy region, Italy. JAMA 2020;323:1574-81.

29 Allen B, El Shahawy O, Rogers ES, et al. Association of substance use disorders and drug overdose with adverse COVID-19 outcomes in New York City: January-October 2020. J Public Health 2021;43:fdaa241.

30 Baillargeon J, Polychronopoulou E, Kuo Y-F, et al. The impact of substance use disorder on COVID-19 outcomes. Psychiatric Services 2021;72:578-81.

31 Algera MH, Kamp J, van der Schrier R, et al. Opioid-induced respiratory depression in humans: a review of pharmacokineticpharmacodynamic modelling of reversal. Br J Anaesth 2019;122:e168-79.

32 Ogungbe O, Akil L, Ahmad HA. Exploring unconventional risk-factors for cardiovascular diseases: has opioid therapy been overlooked? Int $J$ Environ Res Public Health 2019;16:2564.

33 Pourbagheri-Sigaroodi A, Bashash D, Fateh F, et al. Laboratory findings in COVID-19 diagnosis and prognosis. Clin Chim Acta 2020;510:475-82.

34 Chopan M, Littenberg B. C-reactive protein and prescription opioid use. J Nature Sci 2015;1:e122.

35 Orum MH, Kara MZ, Egilmez OB, et al. Complete blood count alterations due to the opioid use: what about the lymphocyte-related ratios, especially in monocyte to lymphocyte ratio and platelet to lymphocyte ratio? J Immunoassay Immunochem 2018;39:365-76. 